\title{
EL TALLER DE TEATRO: UNA PROPUESTA DE EDUCACIÓN INTEGRAL
}

\author{
Gabriel Robles Gavira y Diana Civila de Lara \\ Ayuntamiento de Cádiz , España
}

\section{INTRODUCCIÓN}

La educación como capital en las sociedades meritocráticas y la escuela como uno de los medios para alcanzarlo han convertido esta institución en uno de los pilares fundamentales de las sociedades desarrolladas. Esta importancia se sustenta en el papel de agente socializador y en las funciones que cumple para la sociedad, y que resultan imprescindibles para lograr el progreso social e individual en un mundo que crece en complejidad. La escuela, aunque hablemos de los niveles inferiores, transmite conocimientos, valores y actitudes imprescindibles para la vida en común, abarcando, de forma crasa, los ámbitos político, económico y social.

Observando detenidamente estos tres grandes ámbitos comprobamos que la relevancia de la educación y la escuela es insoslayable.

En la esfera política adquiere significación como instrumento de transmisión, legitimación y búsqueda de consenso en torno al sistema político, el estado, la ideología y los aprendizajes políticos. Desde muy temprano conocemos en que Estado vivimos, ensayamos constantemente su sistema político, comenzamos a conocer nuestros derechos y deberes, nos cohesionamos alrededor de sus símbolos y nos identificamos con hechos y actos de exaltación colectiva, etc.

La función económica resulta la más obvia entre las funciones que se atribuyen a la educación y la escuela. Actualmente su importancia es capital, capacita a los individuos de cara a integrarlos en un sistema laboral y en unos puestos de trabajo cada vez más complejos y volátiles. Se requieren unos conocimientos generales mínimos, tenemos que entender las instrucciones, leer y comprender, conocer cuál es nuestro sistema de medida y cálculo, aparte de otros específicos de cada profesión o puesto que pueden adquirirse a través de una formación especializada, ya sea reglada o no reglada.

Por último, la función social también posee un valor ineludible. En el sistema educativo comenzamos a relacionarnos con otros individuos en un espacio formal, comenzamos a diferenciar distintos roles y posiciones sociales, se establecen relaciones sociales fuera de la familia, aprendemos o reforzamos comportamientos fuera del ámbito familiar, reconocemos otras fuentes de autoridad, integramos normas de comportamiento entre iguales y asimilamos sutilmente lo que se llama currículum oculto ${ }^{1}$ (lllich, 1974), etc.

Estos contenidos, valores y actitudes se incorporan al bagaje del individuo, algunas veces de forma manifiesta otras subrepticiamente, a lo largo de los años de escolarización a través del currículum (asignaturas de historia, lengua, matemáticas, etc.) y la vida escolar (celebraciones colectivas de

\footnotetext{
${ }^{1}$ También Bowles y Gintis, 1985; Dreeben, 1990; Foucault, 1978; Friedenberg, 1963 y Anyon, 1981 en Fernández Enguita, M. Sociología de la Educación; Barcelona; Ed. Ariel; 1.999
} 
identificación nacional o autonómicas, reconocimiento incuestionable del saber y la autoridad jerárquica del superior, en este caso el profesor, aceptación de la disciplina y el orden, fomento de la competencia y la individualización, etc.). Habría que matizar que no solamente a través de la escuela, existen otros agentes de socialización que transmiten estos contenidos, los medios de comunicación, la familia,..., pero es innegable el influjo que ejerce la escuela.

\section{EL TALLER DE TEATRO, ¿COMPLEMENTARIO O FUNDAMENTAL PARA UNA FORMACIÓN INTEGRAL DEL INDIVIDUO?}

El taller de teatro como instrumento para una formación integral de la persona supera muchos de los límites actuales de los métodos de enseñanza.

En primer lugar, se hace necesario reflexionar sobre cómo la escuela ha mutado y se ha ido adaptando a los complejos y vertiginosos cambios que han sufrido nuestras sociedades en los últimos siglos. La educación escolar básicamente sigue igual que cuando surge embrionariamente al hilo del desarrollo de los estados nacionales hasta su posterior reconocimiento como institución social fundamental, universal y obligatoria (Varela, 1991). En ese largo camino desde los siglos XVI y XVII hasta el siglo XXI la imagen de un centro y un aula han cambiado poco. Se podrían hablar de mejoras cuantitativas pero limitadamente de las cualitativas.

Es incontestable que actualmente hay más centros, profesores, alumnos escolarizados y el analfabetismo se ha convertido en un fenómeno residual (González-Anleo, 2002:185-229) ${ }^{2}$. Sin embargo, los centros siguen siendo espacios cerrados a su entorno, con una función principal de custodia, las clases son habitáculos con pupitres que atomizan, inmovilizan e individualizan a los alumnos/as (Varela, 1991:53). El profesor ocupa la clásica posición jerárquica sobre los alumnos/as para facilitar una pedagogía transmisiva, Freire lo llamaba "pedagogía bancaria" (Freire, 1980:75-77), se echa el contenido en las cabezas de los alumnos/as para que después se regurgite en el examen. Y se mantienen, en no pocos casos, los dispositivos sancionadores como medios de normalización y control (Foucault, 1978:175-197). Resulta triste pensar que la educación deba basarse en la pena (exámenes, suspensos, repeticiones de curso, separación y concentración de alumnos/as en guetos escolares, etc.) como único recurso para motivar e incentivar a los futuros ciudadanos de una sociedad "desarrollada". Tonucci (1979) explicaba, que no era buen comienzo si el alumno/a tenía que enfrentarse a dos formas de violencia al ingresar en la escuela, por una parte, se elimina el juego, la curiosidad, la diversión, el descubrimiento y la investigación autónoma del niño/a, y por otra parte, se desprecia su vocabulario, su lenguaje o su expresión corporal.

El taller de teatro o la metodología teatral supera gran parte de estas limitaciones y se convierte en un excelente complemento, iría más lejos, puede significar un elemento fundamental para el trabajo educativo y para la misma vida.

Hay que tener presente que muchos pensadores han reflexionado sobre el hecho de que las personas se comportan como actores que representan roles en la vida cotidiana, Goffman (1971) sistematiza el modelo de la dramaturgia teatral para explicar los comportamientos de las personas en

\footnotetext{
${ }^{2}$ Excluyendo la enseñanza universitaria, actualmente el sistema educativo español arroja cifras importantes en cuanto a centros de enseñanza: 50.018 centros (contabilizando Infantil, Primaria, Secundaria, Bachillerato, Formación Profesional y Enseñanzas de Idiomas, Artísticas y Musicales); profesorado: 525.801
} 
distintas situaciones de la vida. Goffman utiliza el modelo dramatúrgico para interpretar nuestro comportamiento pero el tema del teatro de la vida es antiguo, ya lo tenemos en Calderón de la Barca y Shakespeare.

Por qué reclamamos este protagonismo para el taller de teatro como método. Primero, porque se parte del juego como base de la actividad humana (Huizinga; 1972), es decir, el juego como soporte de una actitud activa y dinámica que facilitará la asimilación de los aprendizajes del taller, partimos del principio de que no se puede realizar bien una actividad que no se haya experimentado. En segundo lugar, porque potencia el desarrollo de la expresión oral y corporal como elementos fundamentales de la comunicación humana. Tercero, porque su metodología es participativa, democrática, fomenta la cooperación, el trabajo en grupo y estimula la reflexión sobre las distintas actividades convirtiéndose en un vehículo para transmitir valores de tolerancia, respeto, solidaridad, crítica y denuncia.

Esta metodología, las actividades que se realizan y los contenidos que se aprenden en el taller nos pueden resultar más útiles para la vida que esa ingente cantidad de información que debemos memorizar durante tantos años de escolarización. Incluso, puede reforzar la formación base y facilitar la asimilación de la formación complementaria que, cada vez más asiduamente, requieren las empresas y los nuevos puestos de trabajo. Los nuevos puestos laborales tienden a ser más versátiles y cambiantes, producto de la paulatina transición en las sociedades desarrolladas del cuestionado modelo fordista (Piore y Sabel, 1990) al postfordista emergente (Coriat, 1993).

En el taller de teatro aprenderemos una serie de destrezas y competencias ricas y fundamentales para la vida cotidiana, en todos sus ámbitos, incluido el omnipresente mundo laboral.

- Fomentar la capacidad para pensar, razonar, criticar o tener iniciativas (por ejemplo a través de improvisaciones, análisis colectivos de las escenas y los ensayos, aportaciones y modificaciones sobre la obra o los ejercicios, etc.).

- Otras competencias estarían relacionadas con la comunicación, debemos leer e interpretar lo leído, hablar con corrección, vocalizar y expresarnos con fluidez (se puede conseguir con lecturas dramatizadas y debate sobre el texto, vocalización, hablar en público, expresión oral y corporal, etc.).

- Otro tipo se encaminaría a la adquisición de habilidades de carácter social, por ejemplo, trabajar en grupos, desenvolverse en las relaciones interpersonales, organizar y planificar el trabajo colectivo y el individual (a través de ejercicios de desinhibición ante el público, dinámicas y trabajo en grupo, coordinación del trabajo de escena y los distintos papeles, diseño, planificación, organización, adquisición y ejecución del vestuario y los decorados, etc).

\section{SINERGIA DE ELEMENTOS (OBJETIVOS, CONTENIDOS Y ACTIVIDADES). HACIA LA FORMACIÓN INTEGRAL A TRAVÉS DEL TALLER DE TEATRO}

Los contenidos del taller se dividen en tres grandes esferas que están relacionadas entre sí, con los objetivos planteados y con las actividades para alcanzarlos. A continuación describiremos cómo logra el taller de teatro alcanzar sus objetivos.

profesores en total; alumnado: 6.783.397 alumn@s (incluyendo los niveles anteriores) y el nivel de 


\subsection{El Juego}

Si lo que buscamos es la formación integral del niño y/o del adolescente, debemos incidir entre otras cosas, en la necesaria inclusión del taller de teatro en la vida del niño y/o adolescente. El taller no pretende hallar o preparar buenos actores, sino proveer al sujeto de una serie de conocimientos, aptitudes y habilidades que mejoren sus relaciones con el medio que le rodea, lo cual se llevará a cabo mediante el juego y de forma lúdica. Alonso del Real y Ferreras Estrada señalan en su libro "Aula de teatro", que el teatro es fundamentalmente un juego; así que el taller de teatro debe convertirse desde el inicio en un foro lúdico ya que además el juego es la base de la actividad infantil y de gran parte de la actividad humana. Como decía Piaget, el juego es una actividad imprescindible, ya que el niño necesita jugar porque esa es su forma de interaccionar con una realidad que le desborda, además el juego sirve para consolidar las estructuras intelectuales a medida que se van adquiriendo. El juego en la actividad teatral no debe entenderse como frívolo o poco serio, sino reflexivo y portador de valores democráticos, de respeto y de cooperación.

\subsection{La Expresión Oral y Corporal}

Desarrollar la expresión corporal para conocer el cuerpo y sus posibilidades como instrumento para su desarrollo personal y como herramienta de conexión con el medio en que se desenvuelve el sujeto. La expresión corporal nos ayuda a interpretar los mensajes corporales enviados por los demás favoreciendo la comunicación interpersonal.

La expresión surge de la dialéctica equilibrada entre la creatividad y la técnica. La técnica reside en un abanico de recursos aportados por el taller de teatro para disponer de distintas posibilidades de actuación ante una determinada situación. Las actividades expresivas son fundamentales para el desarrollo de la capacidad creadora y para los procesos de socialización. Y esta es la razón de que el binomio expresión-comunicación sea uno de los principios en que se fundamenta la educación actual (Motos, T. 2001).

Desarrollar la expresión oral para mejorar la interrelación con el medio y ayudar a una mejor y más positiva resolución de conflictos. Se puede decir que existe un problema siempre que queremos conseguir algo y no sabemos como hacerlo, es decir, los métodos a nuestro alcance no nos sirven. Tenemos una meta más o menos clara y no existe un camino inmediato y directo de alcanzarla viéndonos obligados, por tanto, a elegir una vía indirecta, a dar un rodeo. El sujeto para resolver un problema debe construir una simulación del mismo que le permita considerar las diferentes situaciones del problema, caracterizar estas situaciones de forma que pueda ayudarle a decidir lo que debe hacer, y aplicar los operadores para cambiar una situación en otra. Del tipo de simulación que construya el sujeto dependerá la mayor o menor dificultad que tendrá en encontrar la solución.

En dicha construcción interviene el lenguaje. Se ha comprobado que los sujetos construyen la representación más eficaz del problema cuando este les viene dado de forma directa y sencilla (García Madurga, J. A. y Pardo de León, P., 1999). Por tanto podemos afirmar que a un mayor dominio del lenguaje, obtendremos una mayor comprensión del problema, lo cual nos capacitará para llevar a cabo una resolución más positiva.

analfabetismo se estima que ha descendido hasta el 1,8\% para los varones y el 4,6\% para las mujeres. 
Desarrollaremos esta capacidad en el taller de teatro por medio de la expresión oral y la técnica vocal, motivación a la lectura y posteriores dramatizaciones, etc.

Fomentar la imaginación y la creatividad con el objetivo de dotar al alumno/a de recursos para enfrentarse a situaciones inesperadas y complejas. Esta esfera se alcanzará por medio de la creación y transformación de historias, lo cual, también puede servir para incentivar la lectura. Podríamos acabar con una maravillosa cita de Rodari (2000:12) “...; para quien sabe qué virtud liberadora puede tener la palabra. $<$ <odos los usos de la palabra para todos>>, me parece un lema bueno y con agradable sentido democrático. No para que todos sean artistas, sino para que nadie pueda sea esclavo".

\subsection{Metodología democrática, cooperativa, grupal, reflexiva y crítica.}

Cuando se adopta un estilo de educación democrática, a diferencia de la autocrática o permisiva, estamos apostando por favorecer la adopción de responsabilidades por parte de los hijos. Por el contrario, las autocráticas o permisivas generan dependencia y falta de confianza hacia los padres, por lo tanto, si el taller de teatro es democrático, cooperativo e incentiva la responsabilidad, el respeto y la tolerancia, podemos decir que es un medio idóneo para fomentar dichas actitudes.

El trabajo en grupo y la cooperación se convierten en algo fundamental porque creemos en el taller de teatro como una actividad grupal y democrática. El trabajo en grupo para inculcar una actitud cooperativa entre todos sus miembros. Según los valores que transmitamos iniciaremos el proceso grupal más adecuado a nuestros objetivos. El grupo puede ser portador de valores y actitudes de cooperación y solidaridad, si el colectivo es consciente de esto intentará instaurar otro tipo de relaciones entre sus miembros. Como conseguir esto: a través de juegos y dinámicas que fomenten la participación de todos/as, que cuenten con las necesidades y opiniones de los demás y que genere un espacio de confianza entre todos/as (Amani; 1995:28).

Análisis y resolución de conflictos mediante el juego dramático para que el sujeto construya el problema y le permita considerar las diferentes situaciones del mismo. La realidad debe entenderse en el marco de los elementos que la constituyen y con las relaciones que entre ellos se establecen y que le dan forma (Amani; 1995). Mediante el juego dramático podemos estudiar y experimentar una misma situación desde diversas ópticas lo que nos llevará a una mejor comprensión, análisis y crítica del problema. Siguiendo nuevamente a Rodari (2000:26) diríamos que "Es teatro; meterse en el pellejo de los otros, ponerse en situación, inventarse una vida, descubrir nuevos gestos".

3.4 La experiencia de los Talleres de Teatro Infantil. Centros Socioculturales "La Viña" y "EI Bidón"

La explícita apología hacia este instrumento deviene de la experiencia en los Talleres de Teatro Infantil de la Fundación Municipal de Cultura del Ayuntamiento de Cádiz (España).

La población destinataria de los talleres ha sido un grupo de 24 niños/as/jóvenes de 5 a 12 años de la ciudad de Cádiz, han estado divididos en dos grupos, cada uno de su Centro Sociocultural. La zona moderna de Cádiz, en el Centro Sociocultural "El Bidón" y el casco histórico de Cádiz en el Centro Sociocultural "La Viña". Cada taller ha contado con 12 participantes, que han asistido a una sesión semanal de 3 horas de duración en el caso del Centro Sociocultural "El Bidón" y de 2 horas y 30 minutos en el Centro Sociocultural "La Viña". 
En el caso del Centro Sociocultural "El Bidón" han asistido al taller de teatro 12 niños con edades comprendidas entre los 5 y los 12 años. En este centro se ha iniciado a los participantes al teatro de sombras y a los malabares.

Las diferencias de edad en el taller de teatro ha sido una dificultad añadida. Un ejemplo concreto de la dificultad de adaptar el taller a las distintas edades lo tenemos en que mientras los niños de doce años comenzaban a familiarizarse con malabares que requieren una mayor habilidad y coordinación de movimientos (las bolas o el plato chino), otros niños, mas pequeños, aprendían a manejar el aro, más adecuado a sus edades (6,7 u 8 años). Finalmente, los de 5 años hacían ejercicios de coordinación general (combinación y alternancia de movimientos del tronco y las extremidades) y equilibrio (Martinez de Haro, $\mathrm{V}$. y Hernandez, J. L. 1989), por ejemplo, una actividad fue realizar correctamente volteretas, etc.

Al Centro Sociocultural "La Viña" han asistido 12 niños de entre 5 y 12 años. En este centro se ha iniciado a los participantes en el teatro de sombras.

¿Cómo se han alcanzado los objetivos del taller?. Un ejemplo concreto de este centro lo encontramos en el teatro de sombras. A los más pequeños se les motivó para que descubrieran las sombras, sus colores, su movimiento, sus variaciones en el tamaño, etc. Por el contrario, cuando nos encontramos con la siguiente etapa evolutiva el nivel de exigencia es mayor. Estos participantes elaboraron un guión, se les pedió que utilizaran, además de los títeres que previamente habían elaborado, complementos como puede ser música, luces, etc.

La diferencia en las edades de los alumnos requería un doble esfuerzo. Los participantes de menor edad, que en algunos casos no alcanzaban a leer y a escribir correctamente, se encuentran sumergidos en juegos de ficción, en los que comienzan a poder representar aquello que no está presente. Sin embargo, los de mayor edad podían llegar a desarrollar guiones en colaboración con otros niños/as, participar en juegos de reglas más complejas, etc.

Entre los 2 y los 7 años los juegos irán evolucionando, así, a partir de los 7 años aproximadamente, los niños se atienen a obligaciones aceptadas de forma voluntaria, mediante acuerdos, que son las normas. (Garcia Madurga, J. A. y Pardo de León, P.; 1999:58). Todo ello, nos conduce a la necesidad de sugerir grupos en los que las edades de los participantes sean más homogéneas.

\subsection{Representaciones de los talleres. El examen final.}

Durante el curso, los alumnos/as crearon los guiones de las obras que posteriormente se representaron al finalizar el taller, elaborándolos a partir de técnicas de creación de historias (Rodari, G., 2000) en las que todos participaron y aportaron sus ideas.

Cuando esta creación colectiva se pone en marcha hay que atender a otras tareas. Además de la creación del guión y el aprendizaje de los distintos papeles había que pensar y construir la escenografía de las obras.

La escenografía, por sí sola, aporta todo un campo de aprendizaje. En primer lugar, se intenta reciclar material del taller. Así percibimos por un lado los distintos papeles que hay que realizar para poner en marcha un montaje (elaboración de la escenografía, elección o composición de la música, realización del vestuario...), y por otro lado, evitamos el consumo excesivo, dando importancia a la labor del reciclaje, aprovechando así el material ya existente y fomentando la creatividad e imaginación a la hora de realizarlo. 
Por otro lado, los alumnos/as se entrenan en la labor de distribuir espacios y situar objetos que complementen y apoyen su trabajo en escena. Y, además, el hecho de ir ellos mismos a buscar y comprar los materiales (con la supervisión de la monitora) que necesitaban para la representación fue un extraordinario ejercicio para la vida cotidiana: se relacionaron con otras personas que desempeñan otros roles distintos al maestro, los padres, etc.; calcularon que cantidad de cada material necesitaban ajustándose a un presupuesto muy limitado; eligieron un portavoz, un tesorero; debatieron sobre los colores, las texturas, las calidades, etc; y manejaron un presupuesto (dinero) semiautónomamente (la monitora aconsejaba y supervisaba). Seguramente, esta actividad equivalió en aprendizaje a varios días o semanas en clases y, desde luego, es más difícil de olvidar y más gratificante que una lección memorizada sobre un tema desligado de la vida diaria.

Un caso concreto de participación y motivación en el montaje final fue una alumna de 12 años de edad, del centro sociocultural "El Bidón", que escribió a ordenador el guión aportando un nuevo personaje y su texto.

Además los alumnos de mayor edad diseñan su propio vestuario e incluso la escenografía que más tarde se realizó en común.

Este año el montaje final se llevo a cabo en la Sala Municipal de Teatro "Central Lechera" lo que ha significado un considerable cambio que ha aportado importantes beneficios como son:

- Posibilidad de que los dos grupos expongan su trabajo el mismo día enriqueciéndose uno del otro.

- Acostumbrarse a asistir a actos públicos respetando las normas más elementales de cortesía: respetar a los compañeros que están en el escenario y al público, guardando el debido silencio,...

- Experimentar el hecho de conocer y actuar en una sala de Teatro.

- Familiarizarse con los componentes de una sala de teatro como el escenario, los telones, los focos, etc.

Las representaciones fueron un rotundo éxito, tanto para los chicos/as que actuaron como para los padres y madres que se emocionaron y reconocieron un trabajo serio y bien realizado. $Y$ como broche final, al terminar el acto, se hizo entrega de un diploma y un CD a cada participante que incluía todos los temas utilizados en los dos montajes.

\section{EVALUACIÓN}

La evaluación en el taller de teatro se ha llevado a cabo mediante la observación directa y continua de cada sujeto y su evolución. Utilizando técnicas de evaluación de tipo cualitativo como: verbalización simple, grabaciones, escritura, collage, técnicas participativas (murales, asambleas...), observacionales. A continuación se llevo a cabo un trabajo de interpretación de toda esta información para contrastar los objetivos con los resultados.

Atendiendo a las necesidades de los participantes y haciendo hincapié en sus carencias y aspectos menos desarrollados, se ha intervenido sobre ellos para su adecuada evolución. 
Evaluación de los objetivos del taller:

1- Ampliar el marco de experiencias del niño y/o adolescente. Conocer y relacionarse con otros niños forma parte de este objetivo y durante el curso del taller y mediante le observación directa hemos visto nacer nuevas amistades que ahora mantienen su relación fuera del taller. El niño se enriquece relacionándose con otros niños y experimentando otras formas de expresión como pueden ser el teatro de sombras, la música, etc.

$2^{0}$ Incentivar el trabajo en equipo y la cooperación. Mediante diversas técnicas se ha hecho hincapié en comportamientos no competitivos sino cooperativos y se ha fomentado el que los participantes trabajen en equipo y se organicen autónomamente. A su vez, se han establecido responsabilidades rotativas (encargados del material de maquillaje, vestuario, etc.) y compartidas (de forma que todos sean responsables del taller en general aunque sean encargados de algún material en particular).

3 o Desarrollar la imaginación y la creatividad. Lo cual se ha llevado a cabo mediante técnicas de creación de historias e improvisaciones. Los participantes han llegado a entender perfectamente el término improvisación comportándose paulatinamente de una forma más espontánea y natural. Mediante técnicas de creación de historias los participantes han potenciado su creatividad, imaginando personajes e historias cada vez más originales, complejas y menos estereotipadas.

$4^{\circ}$ Fomentar el reciclaje. Se ha logrado de una forma creativa, reutilizando el material ya reunido. Por ejemplo: los participantes han aportado ropa y objetos que iban a desechar y que en el taller se han transformado en vestuario.

50 Dotar al niño de las distintas técnicas y recursos expresivos para mejorar sus capacidades de expresión-comunicación. Se han utilizado técnicas de expresión corporal y oral (Motos, T. y Tejedo, F., 1999, y Motos, T. y Aranda, L.G., 2001). Gradualmente, se ha tomado conciencia de las potencialidades del cuerpo (el cual expresa y comunica a los demás) y de como podíamos utilizarlo, convirtiéndolo en un recurso que facilitara y enriqueciera el trabajo a la hora de establecer contacto con nuestro entorno. Así mismo, se ha entrenado la voz para que pudiera expresar más allá del puro lenguaje. En este sentido, la vocalización, la entonación, etc., fueron herramientas que se han ido desarrollando y perfeccionando para una mejor comunicación.

\section{BIBLIOGRAFÍA}

HUIZINGA, J. Homo Ludens; Madrid; Ed. Alianza; 1972

CORIAT, B. El taller y el robot. Ensayos sobre el fordismo y la producción en masa en la era electrónica; Madrid; Ed. Siglo XXI; 1993

PIORE, M. J. y SABEL, C. F. La segunda ruptura industrial; Madrid; Ed. Alianza; 1990

GofFMAN, E. La presentación de la persona en la vida cotidiana. Buenos Aires; Amorrortu; 1971

ILLICH, I. La sociedad desescolarizada; Barcelona; Ed. Barral; 1974

Varela, J. y Álvarez-Uría, F. Arqueología de la escuela; Madrid; Ed. La Piqueta; 1991 
FREIRE, P. Pedagogía del oprimido; Madrid; Ed. Siglo XXI; 1980

FOUCAULT, M. Vigilar y castigar; Madrid; Ed. Siglo XXI; 1978

TONUcCI, F. La escuela como investigación; Barcelona; Ed. Avance; 1979

Alonso del Real, G y Ferreras Estrada, J.: Aula de teatro; Madrid; Ed. Akal; 1996.

Motos, T y G. ARANDA, L. Prácticas de la expresión corporal; Ciudad Real; Ed. Ñaque; 1999

García Madurga, J. A. y Pardo de LeÓn, P. Psicología evolutiva; Madrid; Ed. UNEd; 1999.

RODARI, G. Gramática de la fantasía. Introducción al arte de inventar historias; Barcelona; Ed. Del Bronce; 2000

Colectivo Amani. Educación intercultural. Análisis y resolución de conflictos; Madrid; Ed. Popular; 1995.

GonzÁlez-ANLEO, J. Panorama de la Educación en la España de los Cambios; Madrid, Revista Española de Investigaciones Sociológicas, oㅜ 100, octubre-diciembre 2002, pp. 185-229.

FERNÁNDEZ ENGUITA, M. Sociología de la Educación; Barcelona; Ed. Ariel; 1.999. 